\title{
Case Report: Implant Placement and Immediate Loading With Simultaneous Bone Regeneration following Jaw Odontogenic Cyst Enucleation
}

\author{
Lorenzon G1 ${ }^{1}$, Buttarello GM$^{2 *}$ and Chessa $\mathbf{G}^{3}$ \\ ${ }^{1}$ Private Practice, Brandizzo, Torino, Italy \\ ${ }^{2}$ Private Practice, Torino, Italy \\ ${ }^{3}$ Institute of Oral Pathology, School of Dentistry, University of Sassari, Italy
}

\begin{abstract}
A patient with an odontogenic cyst in the lower jaw was referred to and treated at the clinic. Referring to patient needs it was decided to perform implant rehabilitation with immediate loading at time of cyst removal. The cyst was enucleated and three implants were inserted. Also, simultaneous grafting with collagenated porcine bone substitute was carried out in order to accelerate bone healing. X-ray follow-up 11 and 18 months after cyst removal and implant placement, showed good bone regeneration and satisfactory implant and prosthetic rehabilitation.
\end{abstract}

Conclusion: Using one-piece intra-oral welded implants, resulting in low patient morbidity, a low overall prosthetic rehabilitation cost and beneficial outcome was carried out.

Keywords: Odontogenic cyst; Dental implant loading; Immediate; Bone regeneration

\section{Case Report}

A 75-year old female patient in good health reported to our dental clinic with several missing teeth in the maxilla and a post cyst-removal site in the right jaw, a post cyst-removal site in 36 and an odontogenic cyst in region 35 (Figures 1 and 2). Treatment started with a full arch implant procedure in the upper jaw and a provisional prosthetic fixed rehabilitation. Three months later the second surgical procedure was carried out to complete prosthetic rehabilitation in left lower quadrant and to remove the cyst. One-piece and wide-square-threaded implants (Tipo L, Implamed, Cremona, Italy) were chosen in order to ensure self-drilling capacity, good primary stability and large bone-implant surface contact. Flapless surgery and mini-invasive drilling technique were employed to preserve bone in the cyst enucleation site.

Surgical procedure started with 36 root extraction and 35-cyst enucleation (Figure 3), followed by placement of 3 implants in 34, 35 and 36 sites (Figures 4 and 5) with simultaneous grafting by a collagenated porcine bone substitute (Gel 40, OsteoBiol, Pianezza, Italy) in the residual cyst cavity (Figure 6). In the 34 site, the implant was inserted transfixing the cyst cavity to ensure good primary stability by a bicortical engagement (Figure 5 ). Once primary stability assessment was completed, implants were splinted by two $1,5 \mathrm{~mm}$ diameter titanium bars applying the syncristallization technique [13 ], an intraoral welding procedure directed to receive higher primary stabilization, To further strengthen the construction and ensure immediate functional and aesthetic rehabilitation a tripod structure with adequate stability and stiffness (Figure 7) was preferred.

A radiographic examination was performed (Figure 8) and a provisional resin bridge was fabricated. The cemented provisional bridge showed total absence of occlusal contacts in order to avoid any biomechanical overload. Four months after implant placement, abutments were further refined with a tungsten-carbide bur in order to provide a correct matching of peri-implant soft tissues and a final prosthetic restoration (Figure 9) was initiated. Once the final metalceramic bridge restoration was provisionally cemented (Figure 10) the patient was recalled after 3 months for hygiene control, bridge removal and definitive cementation. Eleven months after implant placement, radiography (Figure 11) showed good bone density around implants and good trabecular bone regeneration in the cyst enucleation site. Normal prosthetic function and no clinical symptoms were reported. However, radiography showed a residual radioluscent part close to the apex of the 36 implant (Figure 11) and the patient was consequently subjected to another surgical bone curettage treatment in the recurring cyst site. Again grafting was performed with collagenated porcine bone substitute (Gel40).

At 18 months after first the implant procedure, functional implant osseointegration and optimal bone regeneration where recorded (Figure 12) and the patient reported good function and absence of clinical symptoms.

\section{Discussion}

The case presented required solutions to many clinical questions i.e. enucleation of a cyst in region 35 , residual 36 root extractionand

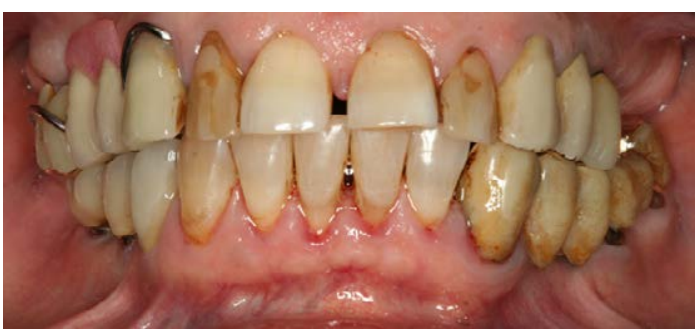

Figure 1: Intraoral photo before treatment.

${ }^{*}$ Corresponding author: Dr. Gian Marco Buttarello, Via Sabotino 12, 10093 Collegno (To), Italy, Tel: +393482314899; E-mail: gianmarco.buttarello74@vodafone.it

Received December 20, 2014; Accepted January 21, 2015; Published January 27, 2015

Citation: Lorenzon G, Buttarello GM, Chessa G (2015) Case Report: Implant Placement and Immediate Loading With Simultaneous Bone Regeneration following Jaw Odontogenic Cyst Enucleation. Dentistry 5: 281. doi:10.4172/21611122.1000281

Copyright: @ 2015 Lorenzon G, et al. This is an open-access article distributed under the terms of the Creative Commons Attribution License, which permits unrestricted use, distribution, and reproduction in any medium, provided the original author and source are credited. 


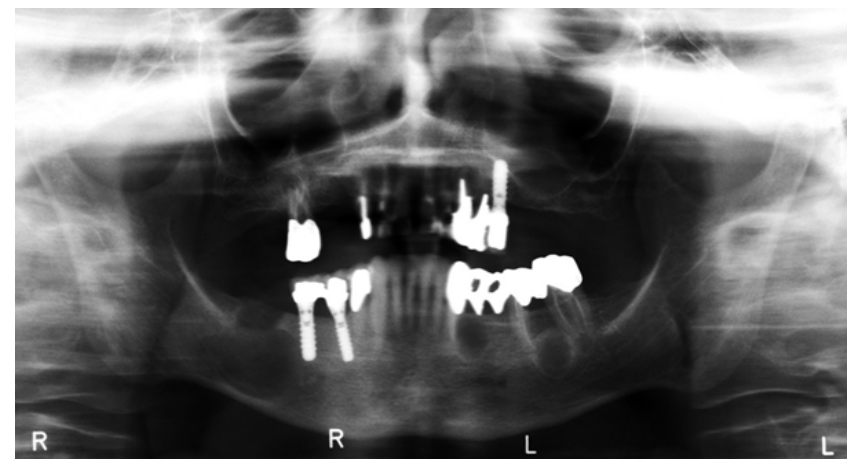

Figure 2: Radiography prior to treatment.

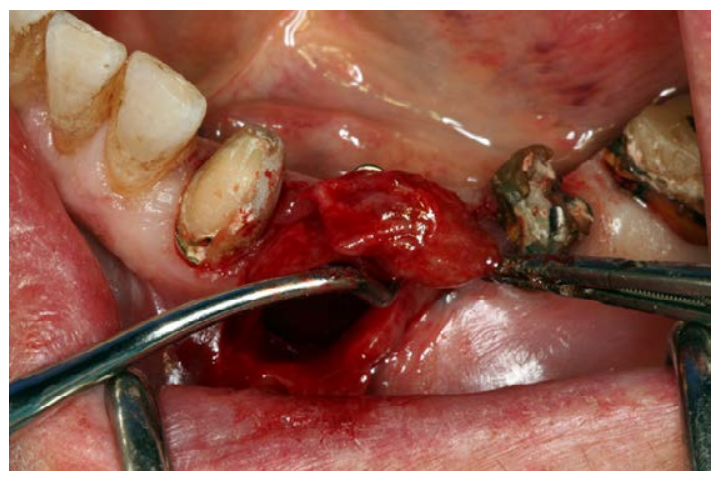

Figure 3: Surgical enucleation of the cyst region 34.

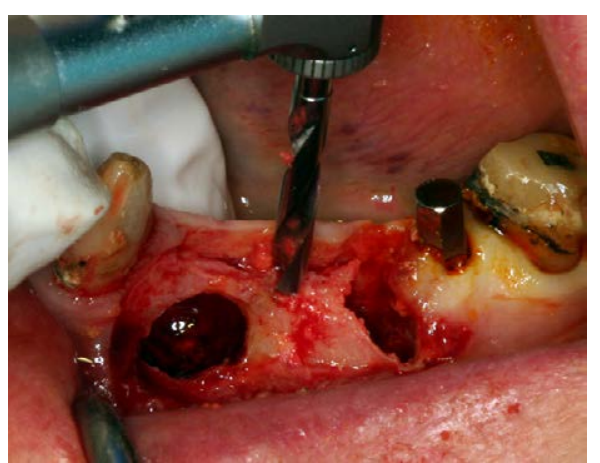

Figure 4: Implant positioning.

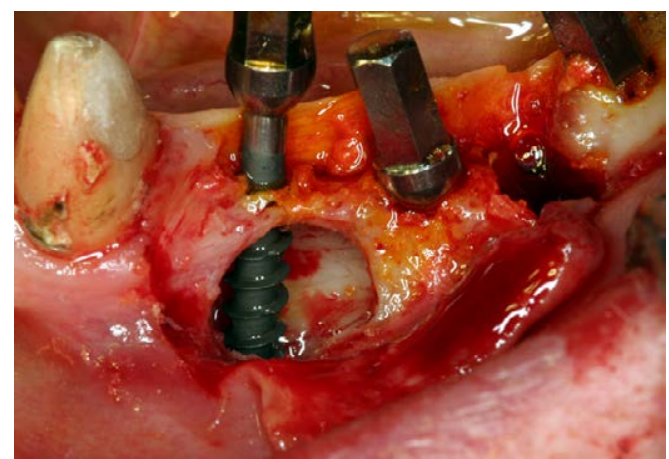

Figure 5: Residual cyst cavity transfixed by implant.

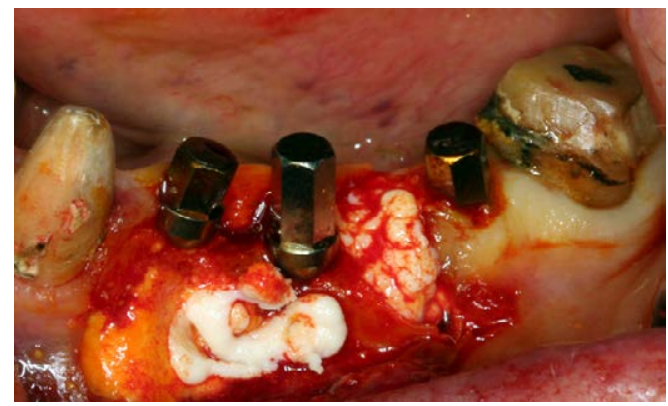

Figure 6: Cyst cavity filled with bone substitute.

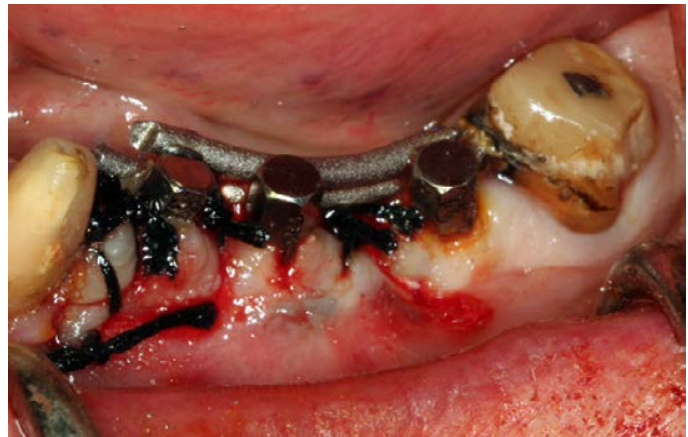

Figure 7: $\mathrm{Ti}$ - bars welded to the 3 implants giving high stability

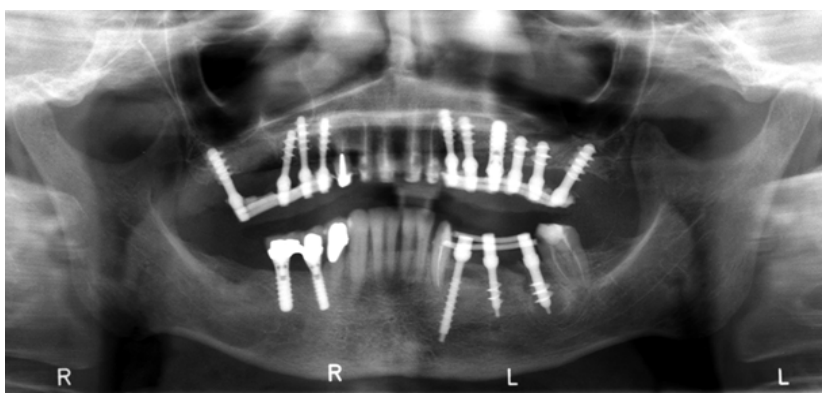

Figure 8: Postop x-ray showing residual cyst cavity filled with bone substitute.

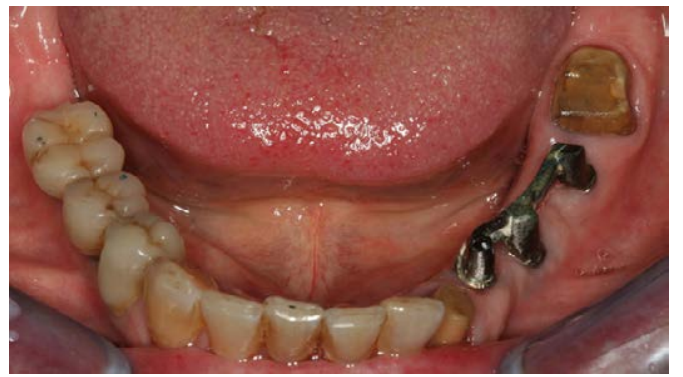

Figure 9: Intraoral image at 4 months, pre-prosthetic.

implant rehabilitation in the left lower quadrant. The use of the reported implant procedure was defined as immediate loading allowing a solution to all the requirements of the patient in one single surgery. The use of one-piece implants to further simplify the procedure has 


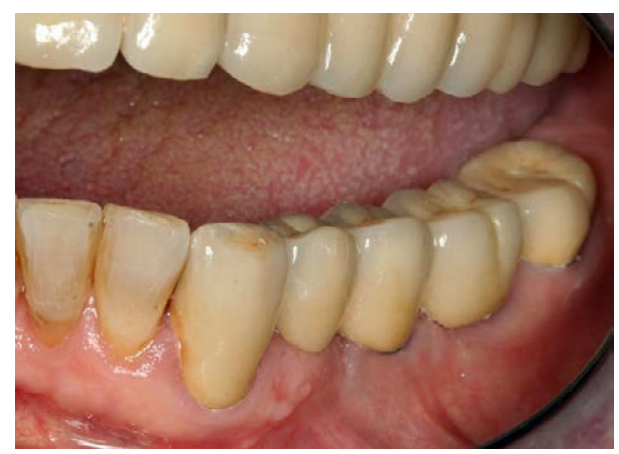

Figure 10: Final restoration, ceramic bridge.

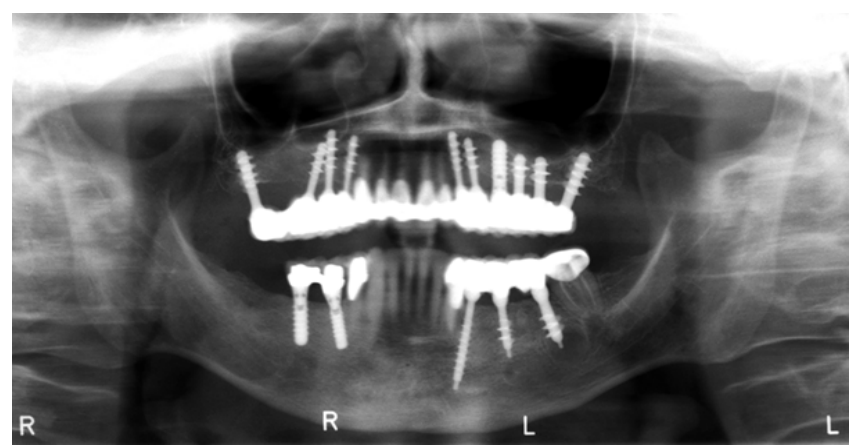

Figure 11: X-ray 11 months postop.

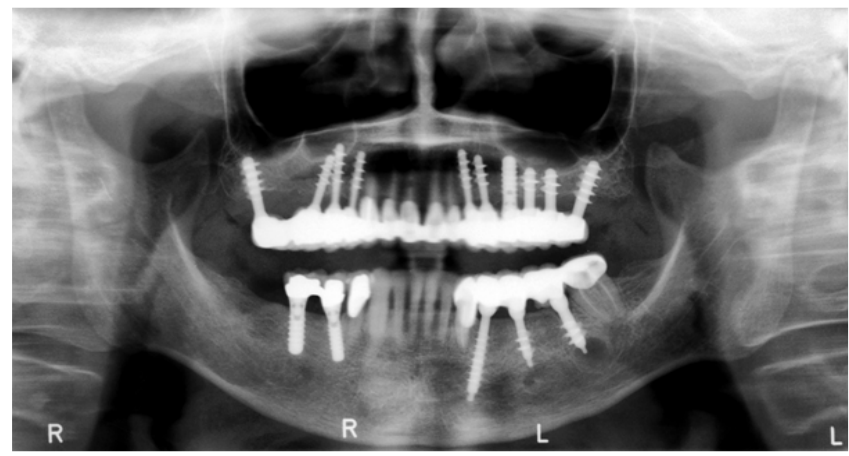

Figure 12: X-ray 18 months postop: residual cyst cavity slowly being reduced. earlier been described being of some benefit [4] and the intraoral welding technique has also shown good results at long-term follow-up [5]. The treatment resulted in good bone regeneration and, as have been reported by others [6], collagenated porcine bone in combination with collagen gel seems to have good ostoeconductive properties without being resorbed too fast. At 18 months of follow-up, both radiographic and clinical examination revealed satisfactory prosthetic rehabilitation as well as ongoing healing of both implants and cystic lesion.

\section{Conclusion}

In conclusion, the advantage of a single surgical intervention, resulting in low patient morbidity, was observed and validated. Furthermore, a reduction in overall prosthetic rehabilitation costs was noted. Long-term success is most likely to be expected; however, further clinical studies should be carried out before this procedure could be extended to other implant rehabilitation cases.

\section{References}

1. Degidi M, Gehrke P, Spanel A, Piattelli A (2006) Syncrystallization: a technique for temporization of immediately loaded implants with metal-reinforced acrylic resin restorations. Clin Implant Dent Relat Res 8: 123-134.

2. Hruska AR (1989) Welding implants in the mouth. J Oral Implantol 15: 198-203.

3. Hruska AR (1987) Intraoral welding of pure titanium. Quintessence Int 18: 683688.

4. Prithviraj DR, Gupta V, Muley N, Sandhu P (2013) One-piece implants: placement timing, surgical technique, loading protocol, and marginal bone loss. J Prosthodont 22: 237-244.

5. Degidi M, Nardi D, Piattelli A (2013) A six-year follow-up of full-arch immediate restorations fabricated with an intraoral welding technique. Implant Dent 22 224-231.

6. Pagliani L, Andersson P, Lanza M, Nappo A, Verrocchi D, et al. (2012) A collagenated porcine bone substitute for augmentation at Neoss implant sites: a prospective 1-year multicenter case series study with histology. Clin Implant Dent Relat Res 14: 746-758. 